\title{
Caractéristiques d'un amortisseur de houle à profils porteurs
}

\author{
M. LEBEY, E. RIVOALEN, J. BROSSARD \\ Groupe d'Energétique et de Mécanique - Université du Havre - 25, rue Philippe \\ Lebon - BP 540, 76058 Le Havre Cedex
}

\begin{abstract}
Résumé
La recherche de l'amélioration de l'efficacitê des amortisseurs de houle est une constante préoccupation aussi bien des ingénieurs qui sont chargés de la protection des structures portuaires ou off-shore, que des chercheurs qui doivent utiliser la houle dans des canaux ou des bassins pour des essais, sans réflexion sur les parois. Après avoir montré l'importance des deux phénomènes conjugués, que sont le phénomène d'interférométrie et la dissipation turbulente, dans le fonctionnement des amortisseurs de type caisson, les auteurs présentent une méthode d'amélioration de l'efficacité de ces types d'amortisseurs. Certe méthode propose l'utilisation d'un dispositif à profilis porteurs en incidence positive par rapport à la direction de la houle. Ces profils ont la propriété d'accentuer la dissipation turbulente tout en participant au phénomène d'interférométrie, l'ensemble concourant à une meilleure efficacité avec un encombrement beaucoup plus faible, ce que montre l'étude expérimentale poursuivie.
\end{abstract}

\section{Introduction}

Depuis longtemps l'homme a été obligé de se préoccuper de la protection contre la houle. Les problèmes qu'elle entraîne sont essentiellement de deux natures: d'une part l'attaque directe des côtes et des suructures portuaires qui provoque leur destruction et d'autre part les perturbations des zones de navigation sous l'effet de la combinaison des houles incidentes et réfléchies, ce qui nuit considérablement à la navigation. Dans les deux cas cela nécessite au moins l'amortissement de la houle si ce n'est sa desiruction complète. Pour obtenir ces résultats deux grandes catégories de dispositifs sont employés: les plages d'amortissement et les caissons qui équipent les digues de protection de ports ou les structures off-shore. Les plages d'amortissement sont des dispositiês qui pour l'essentiel dissipent l'énergie de la houle; elles présentent l'inconvénient majeur d'avoir un encombrement très important incompatible avec les constructions portuaires ou off-shore. Cette difficulté est levée par l'utilisation de dispositifs de type caisson, qui pour l'essentiel reposent sur deux effers conjugués: la dissipation de l'énergie de la houle comme les plages d'amortissement, et la réflexion d'une houle déphasée qui, en se combinant avec la houle incidente, produit un amortissement important. De nombreux travaux ont déjà été effectués pour analyser le fonctionnement et l'efficacité des amortisseurs de houle de type caisson. Quellet et Data ont présenté une synthèse de la majorité des équipements couramment utiissés, parmi lesqueis les caissons, que l'on peut trouver soit sur site réei, soit dans les différents laboratoires. Is ont montré en particulier qu'il est préférable d'utiiiser des installations statiques, et que pour les plages 
d'amortissement, ce sont les profils paraboliques qui sont les plus efficaces. En ce qui concerne les caissons d'amortissement de houle, ils ont mis en évidence l'importance des paramètres de porosité, de rugosité, d'immersion, ou encore les phénomènes $d$ 'interférence. Lhuissier a expliqué l'importance de ce phénomène d'interférence dans le caisson de type Jarlan, phénomène qui semble être d'une importance aussi grande que la dissipation d'énergie dans l'efficacité de l'amortissement de la houle. Comme le précisent Quellet et Data, c'est dans la recherche de l'optimisation de ces deux effets, la dissipation d'énergie et les effets d'interférence, que l'on peut trouver les moyens d'améliorer encore l'efficacité des amortisseurs de houle de type caisson. C'est dans ce cadre que sont présentées ici l'analyse du fonctionnement et les performances d'un nouvel amortisseur de houle de type caisson, conçu à partir de l'utilisation de profils porteurs. Après qu'ait été décrite la constitution de ce nouveau dispositif, l'analyse de son fonctionnement permet de montrer l'imporance de l'optimisation des effets d'interférence et de dissipation sur l'efficacité de cet amortisseur de houle. Enfin ses caractéristiques et ses performances sont comparées à celle du caisson Jarlan déjà utilisé dans de nombreux sites (Port deDieppe, Port de Roscoff, Granville, etc).

\section{Description du dispositif antiréflecteur de boule à profils porteurs et de} l'ensemble expérimental

Comme cela vient d'être rappelé, l'augmentation de l'efficacité des antiréflecteurs de houle de type caisson repose sur au moins deux points: l'accroissement de la diffusion turbulente de l'énergie de la boule et l'optimisation de l'effet d'interférence, avec en plus un impératif de recherche d'encombrement minimal. L'augmentation de la diffusion turbulente peut être obtenue en accroisant la formation des structures tourbillonnaires et en favorisant leur destruction rapide. L'amélioration de l'effet d'interférence peut être obtenu en ajustant d'une part la longueur totale du caisson dans le sens de la houle incidente, et d'autre part les longueurs des différents éléments qui constituent les parties internes du caisson.

Le disposisitif présenté ici est constitué de telle manière que l'amélioration de l'efficacité porte simultanément sur les deux effets: interférence et dissipation d'énergie. Dans la configuration étudiée (voir figure 1), le dispositif anti-réflecteur de houle à profils porteurs se compose d'un ensemble de plans identiques, superposés, orientés avec une incidence négative par rapport à la direction de la houle incidente. Cette superposition de profils porteurs plans est posicionnée devant une paroi verticale plane, en laissant un espace libre entre la paroi de fond et les profils, espace appelé chambre de confinement. Les principaux paramètres qui détinissent ce dispositifs sont présentés sur la figure 1.

Des essais ont été réalisés dans un canal à houle de 3,5 mètres de longueur utile et de 0,3 mètre de large. Le modèle réduit est constinué d'une paroi arrière opaque verticale dont on peut faire varier la position relativement à la paroi composée d'une superposition de volets plans faisant office de paroi semi-réfléchissante. Les paramètres géométriques associés à un tel dispositif sont l'angle d'incidence $\alpha$ des plaques, leur corde l, le pas vertical p et l'encombrement $E$. Cet encombrement $E$ 
du caisson est défini par : $E=1 \cos \alpha+d$. Le nombre de plans est tel que quelle que soit la hauteur d'eau, il y ait toujours des plans qui puissent agir sur la houle.

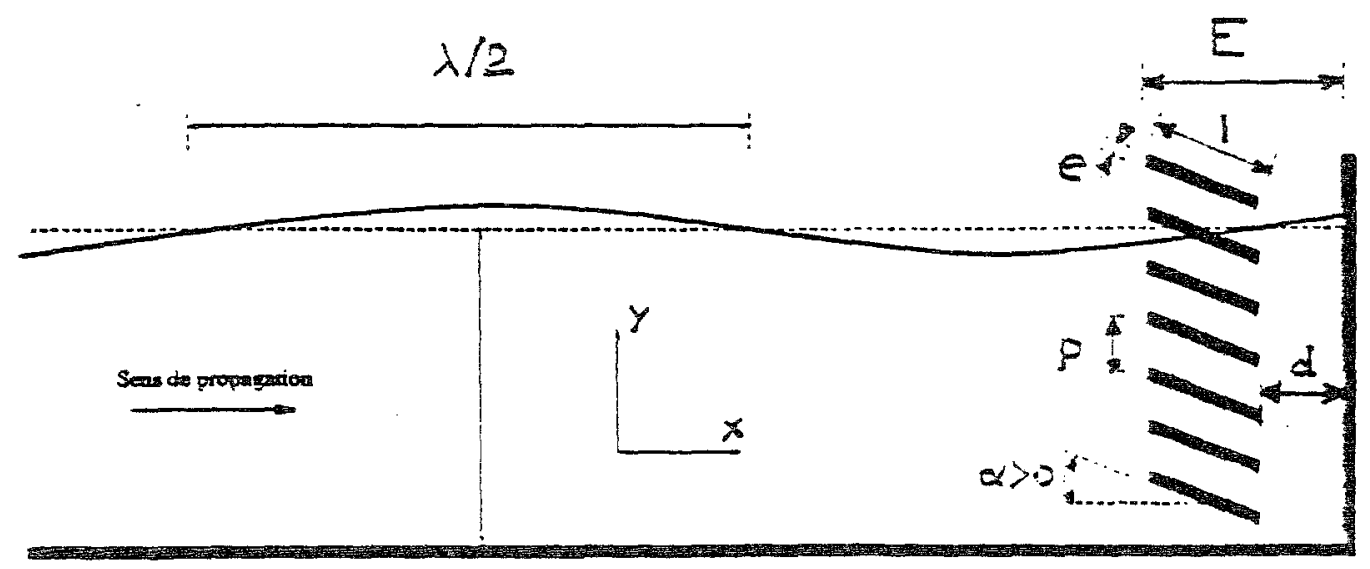

Figure 1: Schéma de l' antiréflecteur de houle à profils porteurs.

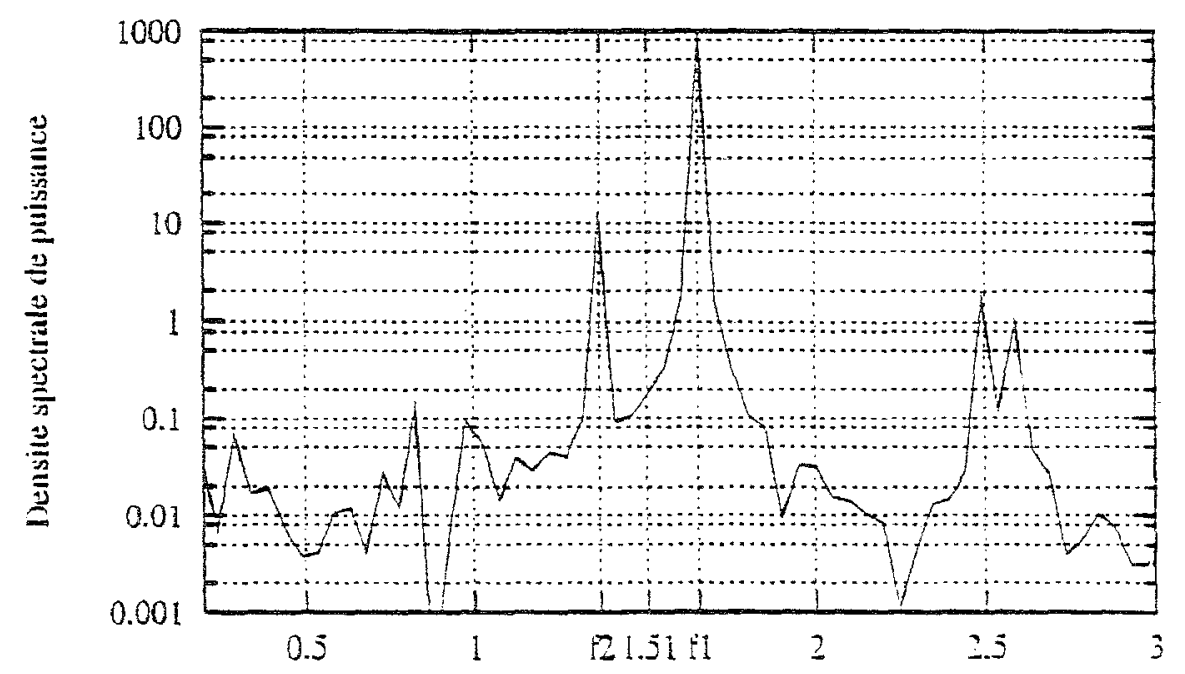

Figure 2: Eremple de spectre de houle qui permer le calcul du coefficient de réflexion. On retrouve les fréquences $f l=1,66 \mathrm{~Hz}$ et $f 2=1.37 \mathrm{~Hz}$

La houle générée devant l'ouvrage est monochromatique de fréquence fixée i $1.51 \mathrm{~Hz}$ pour tous les essais et de longueur d'onde $\lambda$. La hauteur d'eau dans le canal h, est de $0.145 \mathrm{~m}$. En utilisant la relation de dispersion de la houle en profondeur finie on trouve $\lambda=0.61 \mathrm{~m}$. L'amplitude de la houle $\mathrm{H}$ est d'environ $0.09 \mathrm{~m}$. Le calcul du coefficient de réflexion de la houle est réalisé grace au relevé de la position de la surface libre $(\eta(\mathrm{x}, \mathrm{t})$ ) en fonction du temps $t$ (Brossard, Hémon 1994): une sonce résistive fixée sur un chariot se déplace à vitesse constante dans la direction $x$ sur la longueur du canal (1,8 mètres). La signal temporel est earegistré à la fréquence de $200 \mathrm{~Hz}$, pour un échantillon total de 4096 valeurs. Le déplacement de la sonde produit un décallage Dopoler permetant de discréminer les ondes incidentes des ondes réfléchies. Caci est réalisé par une simpie analyse 
spectrale du signal temporel de l'élévation de la surface libre enregistré par la sonde. La figure 2 donne un exemple de spectre obtenu pour une vitesse de sonde de $0.091 \mathrm{~m} / \mathrm{s}$. Dans cet exemple, le premier pic est à la fréquence $f 1=1,66 \mathrm{~Hz}$ et le second à la fréquence $\mathrm{f} 2=1,37 \mathrm{~Hz}$. La fréquence $\mathrm{f} 2$ correspond à la propagation de l'onde incidente à la fréquence du batteur, l'amplitude associée est $\mathrm{A} 2=9,35 \mathrm{~cm}$. La fréquence fl correspond à la propagation de l'onde réfléchie par l'ouvrage avec une amplitude $A 1=1,1 \mathrm{~cm}$. La position des pics sur le spectre peut être donnée simplement par l'équation de dispersion du milieu. Finalement le coefficient de réflexion du mode fondamental de l'onde est donné par la relation : $R(\%)=\frac{A 1}{A 2}=11.7 \%$

\section{Fonctionnement}

Pour mieux comprendre le fonctionnement de l'antiréflecteur de houle à profils porteurs des visualisations ont été réalisées à l'aide de particules de rilsan répandues dans la masse fluide et éclairées avec un plan laser confondu avec le plan de symétrie du caisson; ces particules ont une densité de 1,06. Sur la figure 3 (a et b), on peut voir une reproduction des phénomènes qui prédominent dans le fonctionnement de cet antiréflecteur. En premier lieu, une circulation du fluide apparât autour de l'ensemble de profils porteurs. Cette circulation produit un écoulement moyen descendant dans la chambre de confinement, et un écoulement moyen ascendant sur la face frontal de l'antiréflecteur de houle. Par ailleurs on peut remarquer les tourbillons qui sont produits sur la face frontale et dans la chambre de confinement. Nous verrons dans la suite de la description du fonctionnement, l'importance que prennent cette circulation et ces tourbillons.

Dans le premier cas, comme le montre les reproductions de visualisations, la dissipation turbulente est produite par la présence des profils qui créent d'importants tourbillons sur la face avant et dans la zone de confinement qui sépare les profils de la paroi de fond. Ces tourbillons sont rapidement détruits pour produire de la turbulence Gonc contribuer à la dissipation visqueuse de l'énergie de la houle. Comme on peut le voir sur la reproduction des visualisations (figure 3 ), cette dissipation se produit de deux façons:

1) Au passage du front montant de la houle au travers des profils porteurs (figure $3, a$ ) des tourbillons sont formés sur le bord de fuite des profils. Ces tourbillons se creent avec un déphasage, les premiers à partir du haut. La circulation autour de l'ensemble des profils porteurs les entraîne vers le bas de la chambre de confinement. L'espace réduit provoque leur destruction par intéraction mutuelle et de ce fair la création de turbulence qui conduit à la dissipation visquense.

2) Au passage du front descendant de la houle (figure $3 . \dot{b}$ ), un phénomène similaire se produit. Cette fois-ci, les tourbilions commencent à se former en bas et sont convectés vers le haut par la circulation autour des profils porteurs. Ces tourbillons intérngissent uussi entre eux de manière analogue au cas précécient. ee qui provoque encore la dissipation visqueuse de l'énergie de la houle. 

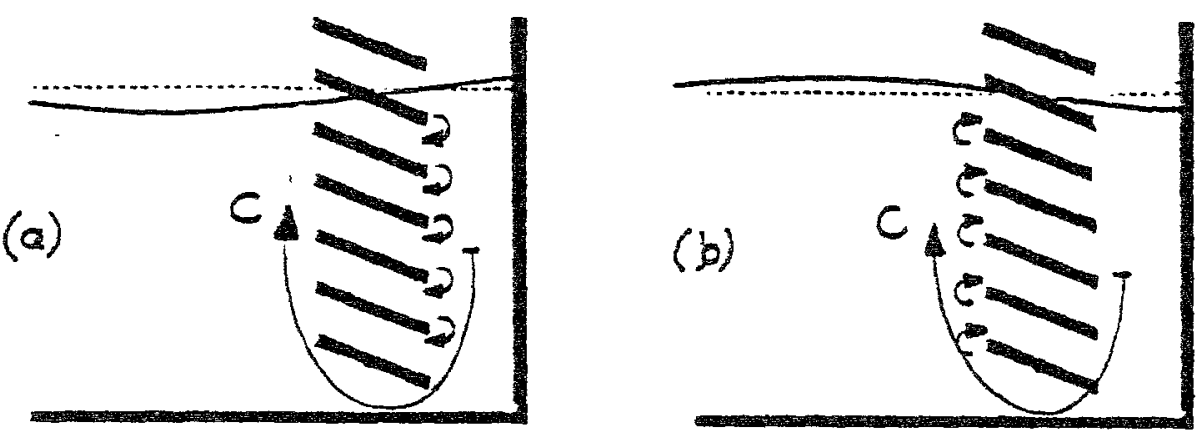

Figure 3: Processus de dissipation dans l'anti-réflecteur de houle à profils: a)formation des tourbililons au passage du front montant, b) formation des tourbillons au passage du front descendant. $C$ indique la circulation moyenne autour de l'ensemble des profilis.

Le second processus qui intervient dans le fonctionnement de l'amortisseur de houle à profils porteurs est le phénomène d'interférence. Comme l'a montré Lhuissier, les dispositifs amortisseurs de houle de type caisson peuvent être considérés comme étant constitués d'une paroi semi-rétléchissante placée à une distance $D$ d'une paroi opaque parfaitement réfléchissante. Dans le cas présent, la paroi semi-transparante est constituée par l'ensemble des profils porteurs. Cet ensemble est soumis à une émission constante d'ondes que constitue la boule incidente. L'onde incidente est partiellement transmise au travers de la paroi semitransparante; cette onde transmise est, quant à elle, intégralement réfléchie sur la paroi opaque puis pariellement réfléchie sur la paroi semi-transparante vers la paroi de fond. I s'étabiit ainsi dans le chambre de confinement un réseau d'ondes. L'ensemble de ces ondes produisent entre elles des interférences qui ont pour conséquence la formation d'une onde réfléchie de beaucoup plus faible amplitude, ce qui provoque la diminution du coeffificient de réflexion de l'antiréflecteur de type caisson, phénomène également présent dans l'antiréflecteur à protils porteurs.

\section{Analvse des résuitats}

Nous avons réalisé une étude systématique pour évaluer l'influence des paramètres p. $\alpha$ et $E$ en mesurant le coefficient de réflexion du mode fondamental émis par le batteur sur le caisson. Le processus résice tout d'abord dans le choix des paramètres constitutifs de la face avant et ensuite dans le positionnement de certe paroi par rapport au fond d.

La particularité de ce dispositif vient ici de l'angle $\alpha$ qui joue un rôle essentiel. Ainsi pour des angles $\alpha$ négatiors le coefficient de réflexion est toujours très important (de 30 a $90 \%$ suivant les valeurs de $p$ et d) (voir higure 4): la iace avant agit très peu sur l'écoulement car dans ces conditions l'incidence telarive des volets par rapport aux lignes de courant reste toujours faible quelle que soit la phase de la houle (en considérant $k<\lambda, l=5 \mathrm{~cm}$ dans tous les essais). La paroi 
composée des profils plans devient transparente face à l'écoulement et n'agit plus ni sur la composition des ondes ni sur la production de tourbillons.

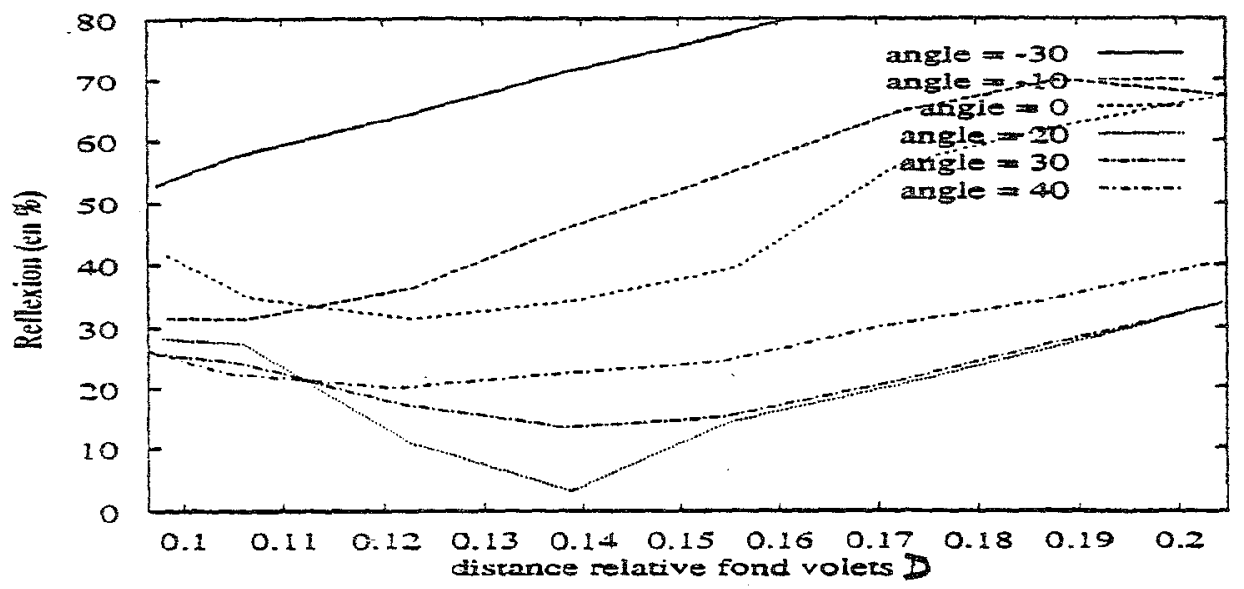

Figure 4: Comparaison du coefficient de réflexion de l'antiréflecteur à profils porteurs, pour des angles $\alpha$ négatifs puis positifs, en fonction de la distance réduite $D=E \lambda$.

Par contre la mise en incidence positive des volets favorise le processus de formation des tourbillons. Sur les courbes donnant le coefficient de réflexion $\mathrm{R}(\%)$ en fonction de l'encombrement réduit $\mathrm{D}=E \lambda$, on retrouve les résultats classiques des caissons: les courbes présentent un minimum du coefficient de réflexion $R$ qui donne l'encombrement optimal $E$ en fonction du pas $p$ choisi et de la longueur d'onde $\lambda$ utilisée (figure 5). L'efficacité du dispositif est optimale lorsque l'on a trouvé le couple $(p, \alpha)$ donnant le minimum de réflexion.

Toutes les courbes présentent une allure analogue (figures $5 a, b$ et $c$ ) avec un miminum du coefficient de réflexion pour une valeur de l'encombrement $E$ variant entre 60 et $100 \mathrm{~mm}$. Les courbes du coefficient de réflexion en fonction de l'angie d'incidence $\alpha$ sont les plus reserrées pour une valeur de l'écartement de $35 \mathrm{~mm}$ (figure $5 c$ ), le plus petit coefficient de réflexion, $5 \%$, étant obtenu pour un angle d'incidence de $35^{\circ}$ at un encombrement $E$ de $70 \mathrm{~mm}$. Pour une valeur du pas $p$ inférieure, égale à $25 \mathrm{~mm}$ (figure $5 \mathrm{~b}$ ), les courbes sont plus étalées avec des coefficients de réflexion plus importants, sauf un cas: $\alpha=20^{\circ}$ et $E=30 \mathrm{~mm}$. Pour une valeur du pas p inférieure, égale à $15 \mathrm{~mm}$ (figure 5a), les courbes sont de nouveau plus espacées, les minima des coefficients de réflexion sont plus importants, supérieurs à $12 \%$.

Par ailleurs, les résuitats ont permis de montrer que le coefficient de réflexion des profils seuls a une valeur de l'ordre $20 \%$ pour des angles compris entre -30 et $30^{\circ}$. 


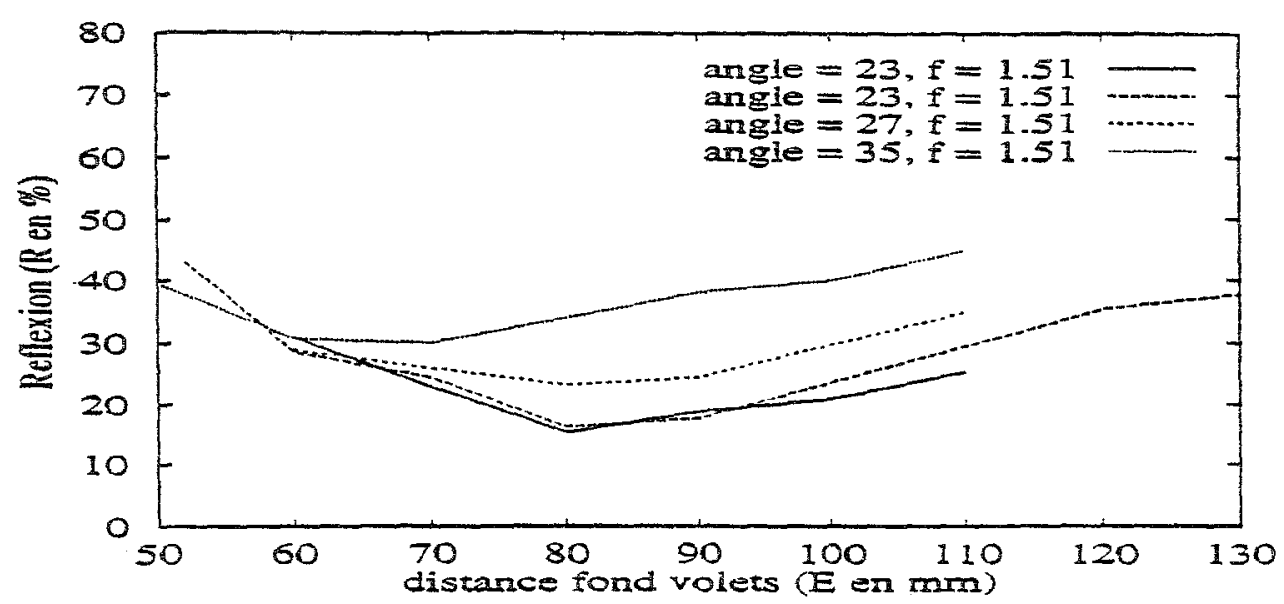

a)

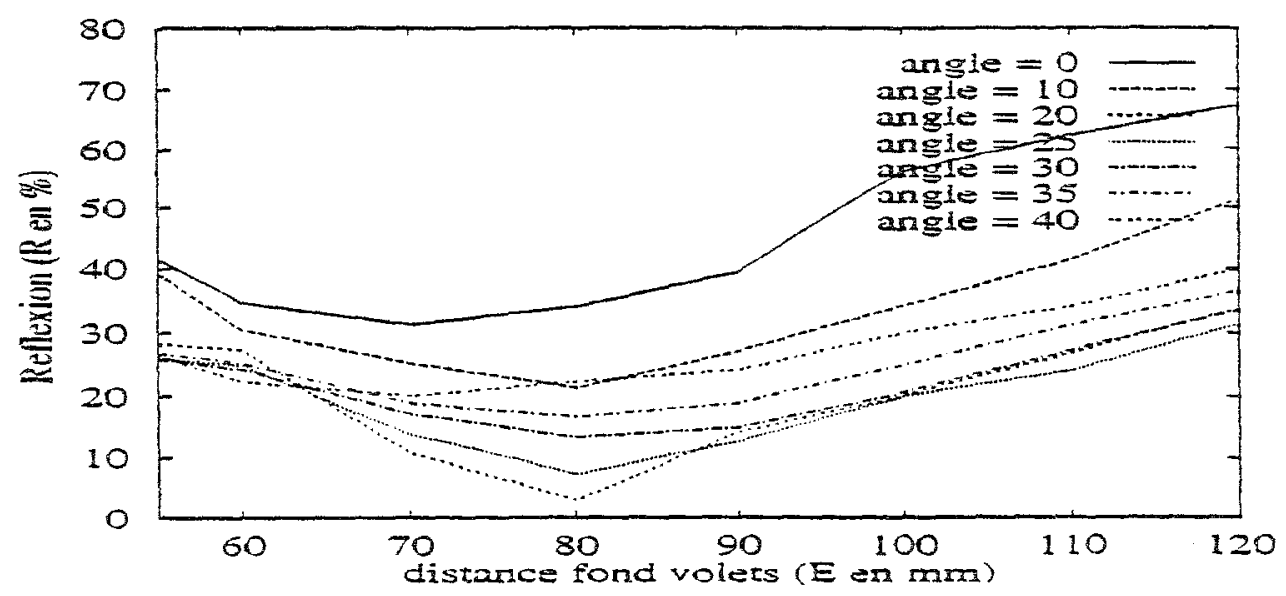

b)

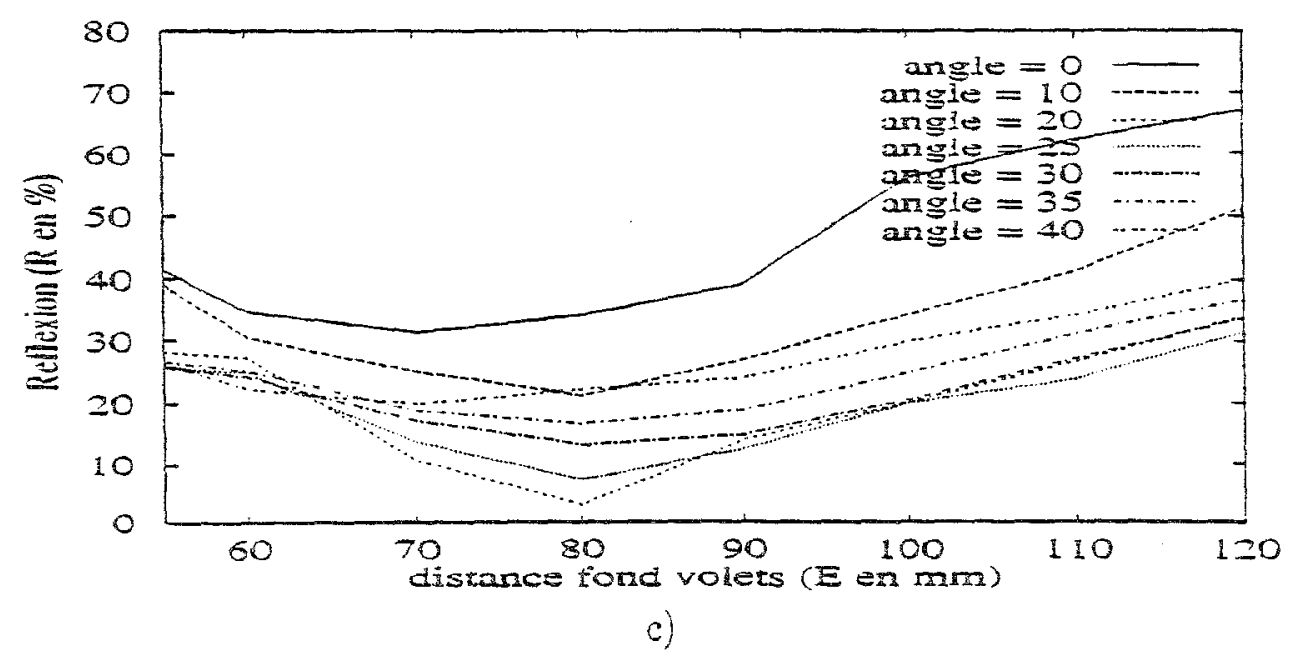

Figure 5: coefficient de réflexion de l'antiréflecteur à profils porteurs, en fonction de l'angles $\alpha$ et de l'encombrement $E$, pour un pas $p$ entre les profils de: a) $15 \mathrm{~mm}$, b) $25 \mathrm{~mm}$, c)35 $\mathrm{mm}$ 
Le dispositif antiréflecteur de houle a donc une éfficacité optimale pour des angles dincidence $\alpha$ des profils compris entre $27^{\circ}$ et $45^{\circ}$. Comme le montre la figure 6, la comparaison de l'efficacité de ce dispositif avec celle du caisson de type Jarlan, obtenue par des essais réalisés dans les mêmes conditions expérimentales, montre que l'encombrement $E$ nécessaire pour ce dispositif est de $35 \%$ inférieur à celui nécessaire au caisson de type Jarlan.

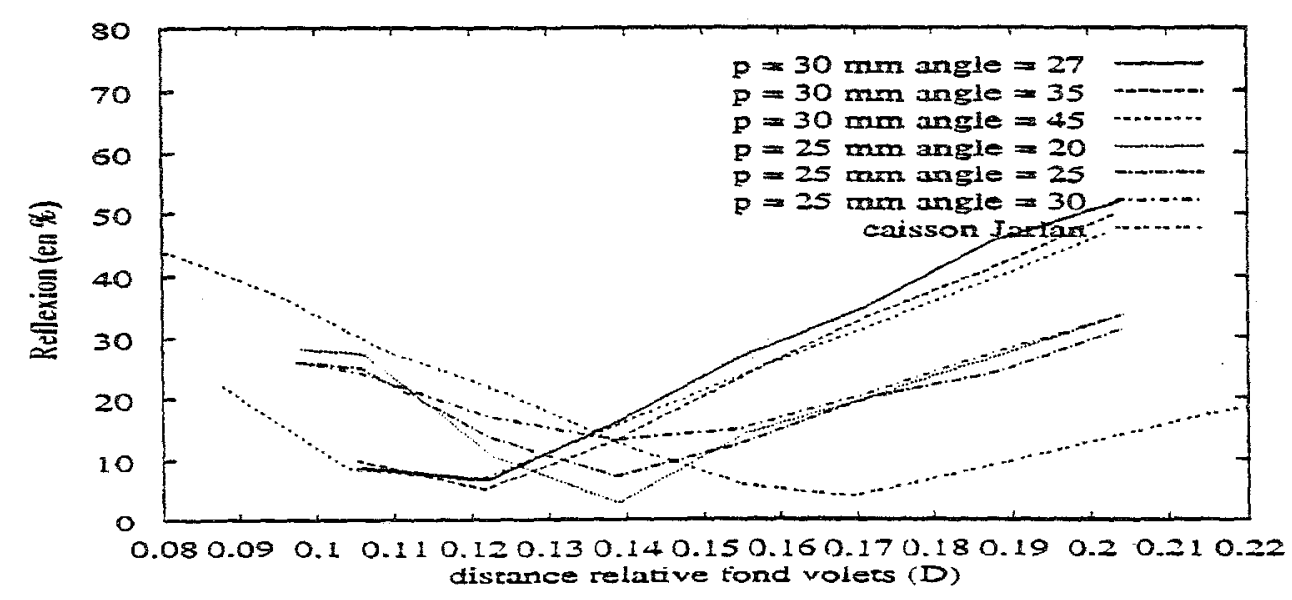

Figure 6: Comparaison de l'efficacité de l'amortisseur de houle à profils porteurs avec celle du caisson Jarlan, essayés dans les mêmes conditions.

\section{$\underline{\text { 5. Conclusion }}$}

L'amélioration de l'éfficacité des absorbeurs de houle de type caisson peut être obtenue en optimisant la combinaison des effets d'interférence et de dissipation turbulente. S'il n'est cependant pas toujours aisé d'obtenir simultanément ces deux résultats, l'utilisation d'un dispositif ì protils porteurs en incidence positive par rapport à la direction de la houle offre cette double possibilité. L'optimisation de ce dispositif est obtenue en ajustant l'incidence des porfils par rapport à la houle et la dimension de la chambre de confinement dans laquelle se produit à la fois la dissipation urbulente de la houle et les phénomènes d'interférence. Les résultats expérimentaux montrent qu'avec un encombrement de $35 \%$ inférieur ce type de caisson offre une efficacité équivalente à celle d'un caisson Jarlan testé dans les mêmes conditions expérimentales. Ce dispositif présente l'avantage d'être adaptable à différentes conditions de houle par réglage de lincidence des profils porteurs; cela permet en particulier de l'utiliser avec des houles de direction non perpendiculaire à la paroi frontale. Il peut donc être avantageusement implanté dans les canaux à houle, bassins d'essais et cuves à turbulence.

\section{Références bibliographiques:}

BROSSARD J. et HEMON A. - Analyse spectraie par effet Dopier de la propagation de la houle - C. R. Acad. Sci. Paris, t. 320, Série II b; P. 171-176, 1995

LHUTSSIER J-F. - Fonctionnement hydrodynamique d'un caisson Jarian - lière Journées Marocaines de Mécaniques - Casablanca 1995.

QUELLET Y. et DATA I. - A Survey of wave absorbers - Joumal of Hydraulic Research, Vol. 24, 1986, $\mathrm{N}^{\circ} 4$ 